$19^{\text {TH }}$ WPA WORLD CONGRESS OF PSYCHIATRY LISBON, 21-24 AUGUST, 2019

\section{Acute stress disorder and implantable cardioverter defibrillator shocks: Three case reports}

BAŞKENT UNIVERSITY

\author{
Department of Psychiatry, Başkent University Faculty of Medicine, Ankara, Turkey
}

\title{
Abstract
}

Implantable cardioverter defibrillator (ICD) therapy is the first-line treatment for the primary and secondary prevention of sudden cardiac death and it is usually well tolerated by the most of patients. Despite the unequivocal medical benefit, treatment with an ICD may also qualify as a potential traumatic stressor, as it may serve as a constant reminder of the underlying cardiac condition. ICD-patients are faced with many challenges affecting their psychological functioning, physical and social well-being and quality of life.

Although the associated factors with distress after ICD implantation are not well understood, studies shows that especially those patients who are younger, have negative beliefs and concerns about ICD and heart failure, type-D personality, a history of psychological distress and receive psychotropic medication are vulnerable to experience anxiety or depression after ICD-implantation. Therefore, patients with ICDs may be prone to experience posttraumatic stress disorder (PTSD) symptoms.

Herein, we report three patients (66-year-old female, 67-year-old male, 45-year-old male) with acute stress disorder (ASD) after ICD shocks. They all well responded to anxiolytic medication therapy.

It is important to predict and identify which patients are at increased risk of developing distress post ICD implantation, as PTSD has been associated with increased cardiac morbidity and mortality in patients with an ICD, and to provide support, to offer appropriate interventions and patient oriented education about patients' fears and concerns.

Key Words: ICD, acute stress disorder, risk factors, PTSD

\section{Introduction}

ICD is an electronic device constantly monitoring heart rate and rhythm, designed to detect and terminate an abnormal heart rhythm. Prior to 2002, most ICDs were implanted for secondary prevention. Currently, most ICDs are placed for primary prevention. Several studies have established the superiority of ICD over anti-arrhythmic medications.

The patient experience of surviving a cardiac arrest or being told about risk for a spontaneous life-threatening arrhythmia resulting in death can certainly be considered traumatic.

The Diagnostic and Statistical Manual of Mental Disorders (DSM -5, 2013) defines PTSD as a trauma-related disorder characterized by symptoms of arousal, reexperiencing the trauma, avoidance, and negative cognitions which cause functional impairment. Posttraumatic stress symptoms experienced within the first month of the traumatic event are classified as ASD.

The best-recognized affective mood states in patients with ICDs are panic disorders, anxiety, and depression, but little is known about their levels of posttraumatic stress. PTSD rates in an ICD clinic have recently been reported approximately $20 \%$.

Herein, we report three patients with ASD after ICD shocks.

\section{Case Reports}

Case 1: A 66-year-old widowed female living alone, with a long standing history of generalized anxiety disorder since her 50s, for which she has been on Sertraline 50mg daily for many years. She has had ICD for 8 years and had received many shocks before. The patient was consulted from the intensive care unit because of the complaints that started after the last shock, 4 days ago, feeling like re-experiencing the event, persistant fear of recieving shock again, problems with paying attention and concentrating, and inability to sleep. Alprazolam $0.75 \mathrm{mg}$ was started in addition to her current treatment and continued for 1 month. After 2 weeks of supportive psychotherapy interviews and with medical treatment, her complaints subsided.

Case 2: A 67-year-old married male, with no past psychiatric history, the consultation liasion psychiatry team was asked to assess the patient for his complaints that started 2 days ago after the ICD storm, anxiety, irritability, exaggerated startle responses and inability to sleep. Alprazolam 0,5mg/day was administered and given for 1 month. His complaints were subsided.

Case 3: A 67-year-old married male, had LVAD and was on the waiting list for heart transplantation, with no past psychiatric history, was consulted from ICU for complaints of fear of being alone, fear of recieving shock again, palpitation, and inability to sleep statrting 3 days ago, after ICD shock. Alprazolam $0,5 \mathrm{mg} /$ day was administered and Sertraline $50 \mathrm{mg}$ daily added after two weeks. His complaints were mostly subsided after 2 months of Sertraline treatment.

\section{Discussion}

An ICD patient is continually exposed to the threat of future defibrillation and must live with a constant visible and tactile reminder of this threat; also faced with many challenges including changes of body image and the expectation of future shocks; all of which have the potential to negatively affect quality of life. Therefore, they may be prone to experience PTSD symptoms.

CBT and pharmacotherapy (with selective serotonin reuptake inhibitors and/or benzodiazepines) combination has been shown to have greater success. Unfortunately, PTSD treatments for ICD patients have not been specifically studied.

Symptoms of PTSD have effect on survival in patients with ICDs. According to recent studies elevated PTSD scores were associated with a 3-fold increased risk of mortality within 5 years compared with ICD patients with no to moderate symptom levels of PTSD, even after controlling for demographic parameters and disease severity. These findings underline the need for routinely applied psychosocial aftercare for patients with ICDs.

The recent guidelines for cardiac pacing and cardiac resynchronization therapy point out that the psychological support of the patient should be one of the main components of successful pacing therapy. To best of our knowledge, no studies have yet reported on ASD in patients with ICD. By early identification and treatment of patients' fears and concers and providing the appropriate support adverse outcomes such as mortality might be prevented.

\footnotetext{
Ladwig KH, Baumert J, Marten-Mittag B, et al. Posttraumatic stres symptoms and predicted mortality in patients with implantable cardioverter-defibrillators: results from the prospective living with an implanted cardioverterdefibrillator study. Arch Gen Psychiatry 2008; 65: 1324-30.

Sears SF, Lewis TS, Kuhl EA, Conti JB. Predictors of quality of life in patients with implantable cardioverter defibrillators. Psychosomatics $2005 ; 46$ (5): $451-7$.

Habibović, M., Denollet, J., \& Pedersen, S. S.. Posttraumatic stress and anxiety in patients with an implantable cardioverter defibrillator: Trajectories and vulnerability factors. Pacing and Clinical Electrophysiology, 2017:40(7);;817-823.

Vilchinsky, N., Ginzburg, K., Fait, K., \& Foa, E. B..Cardiac-disease-induced PTSD (CDI-PTSD): A systematic review. Clinical Psychology Review, 2017:55,;92-106.
} 